\title{
Indústria 4.0: barreiras para implantação na indústria brasileira
}

\section{Industry 4.0: barriers for implementation in the brazilian industries}

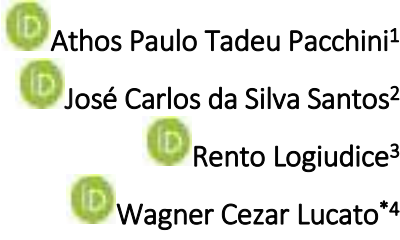

${ }^{1}$ Prof. Dr. Athos Paulo Tadeu Pacchini - Doutorado em Engenharia de Produção pela Universidade Nove de Julho - UNINOVE. Professor do Departamento de Exatas da Universidade Nove de Julho - UNINOVE.

${ }^{2}$ Prof. Dr. José Carlos da Silva Santos - Doutorado em Engenharia de Produção pela Universidade Nove de Julho - UNINOVE. Professor do Departamento de Administração da Universidade Nove de Julho UNINOVE.

${ }^{3}$ Prof. Dr. Rento Logiudice - Doutorado em Engenharia de Produção pela Universidade Nove de Julho - UNINOVE. Professor do Departamento de Administração da Universidade Nove de Julho UNINOVE.

${ }^{4}$ Prof. Dr. Wagner Cezar Lucato* - Doutorado em Engenharia de Produção pela Universidade Metodista de Piracicaba. Professor e Pesquisador do Programa de Pós-Graduação em Engenharia de Produção da Universidade Nove de Julho - UNINOVE. (*) Autor correspondente.

\begin{abstract}
Resumo
Nos anos recentes, a grande inovação em processos tem sido a denominada Indústria 4.0, tida como tão impactante quanto a substituição das carroças pelo automóvel no início do século XX. O termo "Indústria 4.0" tem sido considerado como a Quarta Revolução Industrial. Essa nova tendência utiliza os conceitos da internet das coisas (IoT) aliada à automatização industrial, que agrega inteligência à manufatura e um universo de possibilidades para diferentes fabricantes, produzindo de acordo com os requisitos dos clientes. Este artigo visa identificar as barreiras para a implementação da Indústria 4.0 no Brasil, utilizando como base alguns agentes participantes da cadeia de suprimentos da indústria automotiva. Este setor foi escolhido, pois uma de suas características é a de aplicar constantes inovações em sua cadeia produtiva, buscando o melhoramento contínuo da gestão de seus processos. Por meio de estudos de casos, foram feitas entrevistas presenciais semiestruturadas, com algumas empresas e profissionais atuantes na cadeia automotiva. As entrevistas foram realizadas com gestores das áreas de produção e tecnologia da informação em duas montadoras de automóveis e em um fornecedor de primeira camada. Incluiu-se também nesse estudo uma empresa de consultoria na área de segurança da informação que atua naquele setor e um especialista em Indústria 4.0 que atua na área acadêmica, pesquisando a indústria automobilística. Como conclusão deste trabalho foi possível identificar um conjunto das principais barreiras a serem consideradas para a maior eficácia da implementação da Industria 4.0 nas indústrias.
\end{abstract}

Palavras Chaves: Indústria 4.0. Cadeia de suprimentos. loT.

\begin{abstract}
In recent years, the newest innovation in manufacturing processes has been the so-called Industry 4.0. It has been considered as the Fourth Industrial Revolution, using concepts of like Internet of Things (IOT) combined with industrial automation, which adds intelligence to manufacturing and a myriad of possibilities for industries producing according to customers' requirements. This article aims to identify barriers to implement Industry 4.0 in Brazil, considering some companies belonging to the automotive supply chain. This sector was chosen because it applies constant innovations in its productive chain, seeking the continuous improvement in the management of its processes. Through multiple case studies, personal semi-structured interviews were conducted with some companies and related professional in the automotive supply chain. The interviews were conducted with production and information technology managers at two automobile assemblers and at a first-tier supplier. Also included in this study was a consulting firm in the area of information security that operates in the automotive sector and a specialist that works in the academic area, with research interests in the auto industry. As a conclusion of this work, it was possible identify a set comprising the main barriers that should be considered for the improved effectiveness of the Industry 4.0 implementation.
\end{abstract}

Key words: Industry 4.0. Supply chain. IoT. 
1 Introdução

A quarta revolução industrial já é uma realidade. As revoluções são rápidas, disruptivas e destrutivas, e não têm volta. A Indústria 4.0 tem se mostrado uma resposta aos desafios competitivos que a Indústria enfrenta na atualidade. (Khan, \& Turowski, 2016).

Desde o início da industrialização, em meados do século XIX, avanços tecnológicos levaram a mudanças de paradigmas. Foram as chamadas "revoluções industriais". A primeira, com o uso da energia a vapor quebrou o paradigma da produção artesanal. Com o advento da energia elétrica, no final do século XIX, surge a segunda revolução industrial. Com a cibernética e a digitalização generalizada, veio a 3a revolução industrial. Atualmente, a combinação de diversas tecnologias, internet e os chamados objetos inteligentes (máquinas e produtos), apontam para uma nova revolução industrial, a chamada Indústria 4.0 (I 4.0), apresentando novos paradigmas para a indústria de manufatura (Lasl et al. 2014; Schwab, 2016).

Segundo Dilberoglu et al. (2017) e Mosterman e Zander (2015) a I 4.0 envolve um conjunto integrado de sistemas de produção inteligentes e tecnologias de informação avançadas baseadas em conjuntos de sistemas de softwares integrados.

Assim, a | 4.0, está fazendo com que pessoas, coisas, processos, serviços e dados estejam conectados em rede. São os chamados objetos inteligentes equipados com atuadores e sensores, com códigos QR, sigla do inglês "Quick Response" e chips RFID, sigla do inglês "Radio-Frequency Identification" que tomam decisões, ao longo de toda a cadeia de valor, desde o desenvolvimento de produtos até o serviço ao cliente. Muitas oportunidades já estão se apresentando com esses novos desenvolvimentos, acompanhadas de muitos riscos e desafios para a economia, bem como para as empresas. Além dos problemas de privacidade, proteção e segurança de dados, já são exigidas novas qualificações para as pessoas e novos requisitos para as indústrias (Buhr, 2015; Bauters et al., 2018).

A nova visão da internet, ligada à Indústria 4.0, chamada Internet das Coisas (IoT), está conectando por radiofrequência bilhões de objetos "coisas", tais como sensores, monitores, dispositivos de identificação, máquinas, em uma escala que ultrapassa o uso da Internet tal como tem sido empregada até agora (Dutton, 2015).

O termo "Internet-of-Things" (IOT) foi introduzido pela primeira vez por Kevin Ashton em 2009, para descrever a utilização da identificação por rádio frequência (RFID) e outros sensores para objetos do cotidiano se comunicarem por meio da internet. Ao longo do tempo, o termo evoluiu para um que descreve a loT como uma rede de entidades que estão conectadas por meio de qualquer forma de sensor, permitindo que essas entidades conectadas à internet, sejam localizadas, identificadas e até mesmo operadas. (Ng, \& Wakenshaw, 2017).

Como a loT desenvolveu-se recentemente, ela passou a ser utilizada no Gerenciamento da Cadeia de Suprimentos (SCM), proporcionando uma Cadeia de Suprimentos Inovadora (SCI). Isto trouxe para as companhias oportunidades de melhoria sobre o modelo tradicional, aumentando suas vantagens competitivas no ecossistema empresarial (Li e Li, 2017). A crescente conexão de dispositivos no mundo industrial permitiu que a International Data Corporation (IDC) prevê que o mercado mundial de loT alcance US \$1,7 trilhão em 2020. (Wylie, 2016) 
Já de acordo com Xu et al. (2013), a loT fornece uma promessa de oportunidade de se construir sistemas e aplicações industriais poderosos, alavancando a crescente ubiquidade de RFID, conexões sem fio (wireless), celular e dispositivos de sensoriamento, corroborando a tendência de uma ampla gama de aplicações.

Uma empresa que não lida com os desafios tecnológicos, tem dificuldades de reduzir custos e apresentar novos produtos e/ou serviços, o que a coloca em posição desfavorável em relação a concorrentes que investem pesado em tecnologia (Khan, \& Turowski, 2016). Devido a processos cada vez mais digitalizados e a um crescimento exponencial de dados sensíveis, as cadeias de abastecimento também são impactadas pela | 4.0 (Fanti, 2017).

Segundo a CNI, o desenvolvimento da Indústria 4.0 no Brasil depende em todos os sentidos de maior conhecimento por parte das empresas dos ganhos da digitalização. Com um maior acesso à informação haverá menos incerteza e uma mudança cultural na empresa (CNI, 2016).

Também a CNI, em pesquisa nacional recentemente realizada, concluiu que dentre as empresas consideradas $48 \%$ indicaram utilizar pelo menos uma das 10 tecnologias fundamentais que suportam a | 4.0 (CNI, 2016). Tal afirmação poderia indicar que há, no Brasil, uma certa predisposição das indústrias em geral favorável à adoção da | 4.0. No entanto, isso está longe da verdade na medida em que CNI (2016) indica que no país a I 4.0 ainda é um objetivo de médio e longo prazo para as grandes corporações e um conceito quase totalmente desconhecido pelas pequenas e médias empresas.

Diante desses fatos, surge como lacuna de pesquisa a ser explorada pelo presente trabalho a identificação das principais barreiras enfrentadas para a adoção da I 4.0 nas indústrias brasileiras. Por isso, o objetivo central deste trabalho foi identificar as barreiras mais perceptíveis, neste momento, para a implementação da Indústria 4.0 no Brasil, utilizando como base alguns agentes participantes da cadeia de suprimentos da indústria automotiva.

Para isso, realizou-se uma pesquisa bibliográfica para identificar como a literatura atual trata do referido tema, o que foi complementado por meio de estudos de casos. Para o levantamento das informações no campo, foram realizadas entrevistas presenciais semiestruturadas com os responsáveis pela análise e implementação desta nova tecnologia, em empresas conexas à cadeia de suprimentos automotiva no Brasil.

A escolha do setor automobilístico se deu pelo fato de ser um setor bastante relevante na indústria brasileira (Lucato et al. 2012) e ter também como característica estar na vanguarda da adoção das novas tecnologias (Vanalle, \& Salles, 2011; Mendes, Siemon, \& Campos, 2017), dentre as quais a Indústria 4.0. Nesse sentido e corroborando a escolha feitas pelos autores deste trabalho, o Gerente de Projetos Futuros da Ford do Brasil afirma que a grande inovação tecnológica para os próximos 5 anos, com relação ao produto serão os carros autônomos e com relação aos processos será a Industria 4.0 (Fanti, 2017). Nessa mesma linha, o diretor da Engenharia da Manufatura da Volkswagen do Brasil afirmou que:

A indústria 4.0 é um caminho sem volta que garante a competitividade para o futuro. A empresa que não aderir aos seus conceitos, não será capaz de ser competitiva futuramente. Esta tecnologia permitirá tomar decisões extremamente rápidas, assertivas, detectar problemas, evitar falhas e reduzir custos. (Volkswagen do Brasil, 2017, p. 4).

\section{Fundamentação Teórica}


No início de implementação dos princípios da Indústria 4.0 , as principais tentativas que moldaram as ideias e ferramentas por trás desta nova abordagem, sempre acabavam se referindo ao conceito de máquinas inteligentes que poderiam se comunicar entre elas e com o ambiente (Dopico e al., 2016).

Avanços na comunicação sem fio, em computação e em dispositivos e sensores, juntamente com a redução de custos dessas tecnologias, estimularam e aceleraram o desenvolvimento de sistemas cyber físicos que adotaram o paradigma da Internet das Coisas. A adoção desses sistemas ainda enfrenta várias barreiras que a comunidade de pesquisa e as indústrias estão tentando ativamente superar. Por um lado, os desafios do desenvolvimento estão relacionados, principalmente, a segurança, robustez, disponibilidade, desempenho adequado e otimização do consumo de energia. Por outro lado, o uso desses sistemas produz grandes quantidades de dados refinados que precisam ser processados e inter-relacionados. (Ochoa, Fortino, \& Fatta, 2017)

O aumento explosivo do número de dispositivos conectados à Internet das Coisas (IoT) e o aumento exponencial do consumo de dados apenas refletem como o crescimento do Big Data se alinha perfeitamente à IoT (vide Figura 1). O gerenciamento do Big Data em uma rede em contínua expansão dá origem a preocupações não triviais relacionadas à eficiência da coleta de dados, seu processamento, análise e segurança. (Ahmeda et al., 2017)

Figura 1: Evolução dos objetos conectados

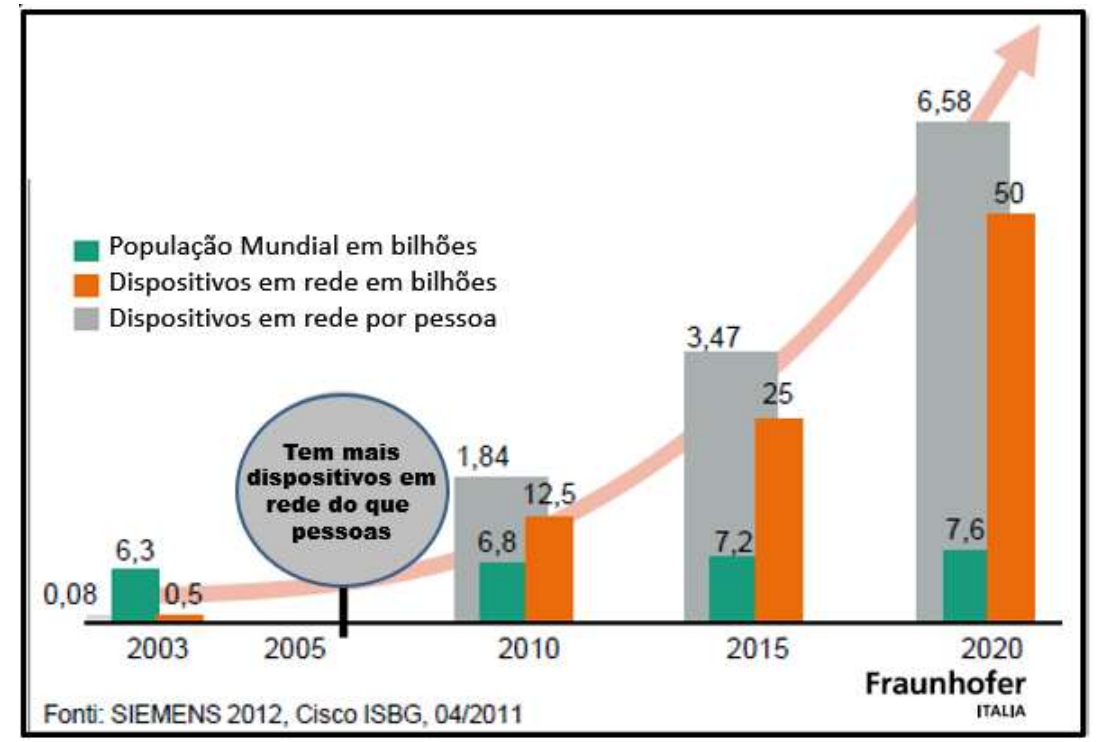

Fonte: Matt, 2014.

A indústria inteligente exige a integração on-line de fornecedores, máquinas e clientes. Uma das dificuldades para isso está nas informações e na comunicação que, se não forem padronizadas, poderão gerar uma falha de interpretação que comprometerá todo o processo de produção. Além disso, não se deve menosprezar as barreiras impostas pela qualificação da mão de obra, uma vez que, a princípio, o trabalho manual é substituído pela programação e monitoramento de máquinas, envolvendo atividades mais analíticas e exigentes. Essa mão de obra precisa estar capacitada e desenvolvida para os novos padrões (Menna et al. 2016).

De acordo com Khan e Turowskl (2016), as principais barreiras que a indústria de manufatura enfrenta são a integração da enorme quantidade de dados heterogêneos, gerados pela interconectividade de todo o 
processo, a flexibilidade de processos produtivos, para se adaptarem a customização da Indústria 4.0 e a segurança como uma das principais preocupações em função da escalada de dispositivos conectados.

Essas máquinas interconectadas conversam e trocam comandos entre si, armazenam dados na nuvem, identificam defeitos e fazem correções sem precisar de ajuda: são as fábricas inteligentes. Apesar das empresas implementarem fortes medidas de segurança, por meio de diversos firewalls, pessoas mal-intencionadas usam algumas lacunas na segurança para cometer os cybers ataques. Segundo Valverde (2015), a cadeia de suprimentos não fica fora desses riscos. "O risco de segurança cibernética só aumentará, pois, cada dispositivo pode ser um ponto de entrada para violações de dados", explica o diretor de Tendências Emergentes da Allianz Global Corporate \& Specialty (AGCS) (IPESI, 2016, p. 1).

O conceito de Indústria 4.0 gera novas categorias de riscos devido ao aumento da vulnerabilidade e ameaças. A conexão do cyber espaço, fabricação sofisticada com o uso de tecnologias, a terceirização de serviços são os principais fatores para aumentar a vulnerabilidade. Assim, a questão importante é como proteger o sistema de fabricação contra cyber ataques, perda de integridade de dados ou problemas com a disponibilidade de informações. (Tupa, Simota, \& Steiner, 2017)

No entanto, o posicionamento das pessoas dentro da produção e as tarefas que devem ser realizadas estão mudando. As tarefas físicas são menos relevante. A experiência no campo das novas tecnologias de comunicação, em termos de planejamento, execução e processos de tomada de decisão, controle ou programação ou na correção de falhas e erros estão se tornando cada vez mais importantes. Além disso, a demanda de conhecimentos e habilidades interdisciplinares para entender o trabalho se tornam mais importantes para a mão de obra envolvida no processo (Gabriel, \& Pessl, 2016).

Toda tecnologia de ponta envolve altos investimentos, porém, no Brasil, não se têm notícias de investimentos governamentais para as indústrias nesta direção. Para competir globalmente, a indústria nacional deve aumentar sua produtividade e sua participação na economia brasileira. "As empresas precisam fazer uma transformação digital tanto em hardware quanto em software para ter uma integração completa de todos os processos" (Exame, 2017, p. 1)

Na Alemanha, por exemplo, o governo federal reservou 200 milhões de euros para subsidiar a "Indústria 4.0". Sua visão: ajudar as empresas a aproveitar a inteligência gerada pela loT para otimizar os processos, aumentar a eficiência e estimular uma maior inovação. Nos Estados Unidos, uma coalizão patrocinada pelo governo formou-se com um objetivo semelhante. A Smart Manufacturing Leadership Coalition (Colisão para a Liderança da Manufatura Inteligente) se concentrou na implementação de "melhores práticas de fabricação inteligente do século XXI". As principais são: Aplicar uma arquitetura de referência que permita uma colaboração e integração perfeitas entre Tecnologia da Informação (TI) e Tecnologia Operacional (OT) (Hannah, 2015).

Como se demonstra, a IOT já está tendo um impacto significativo sobre a fabricação e outros vários segmentos. Além do impulso positivo em várias indústrias, a necessidade de padronização pode dificultar o avanço da implementação de IOT devido a mercados concorrentes. Ainda, até o momento não está claro se esses padrões necessários serão determinados pelo mercado consumidor, pelos líderes do setor de tecnologia, pelas organizações privadas ou regulação geral. Com base nas tendências do passado, a Lei tem falhado 
consistentemente para acompanhar a tecnologia em questões como cyberbullying, proteção de dados, e até mesmo a regulamentação da Internet (Kester, 2016).

Resumindo, no Quadro 1 estão mostradas as principais barreiras identificadas na literatura atual para a implantação da | 4.0 .

Quadro1 - Barreiras identificadas na revisão de literatura

\begin{tabular}{|l|l|l|l|l|l|l|l|l|}
\hline ARTIGOS & ATC & ESC & INV & IOP & EEL & TEC & FOR & REG \\
\hline Ochoa, Fortino, \& Fatta, 2017 & $X$ & & & $X$ & $X$ & $X$ & & $X$ \\
\hline Ahmeda et al. 2017 & & & & $X$ & & & & \\
\hline Menna et al. 2016 & & $X$ & & $X$ & & $X$ & $X$ & \\
\hline Khan, \& TurowskI (2016) & $X$ & & & & & $X$ & & \\
\hline Valverde et al.2015 & $X$ & & & & & $X$ & & \\
\hline IPESI, 2016 & $X$ & & & & & & & \\
\hline Weber, \& Studer, 2016 & $X$ & & & & & & & $X$ \\
\hline Tupa, Simota, \& Steiner, 2017 & $X$ & & & & & & & \\
\hline Wylie, 2016 & $X$ & & & & & & & \\
\hline Gabriel, \& Pessl, 2016 & & $X$ & & & & $X$ & & \\
\hline Exame, 2017 & & & $X$ & & & & & \\
\hline Hannah, 2015 & & & $X$ & $X$ & & & & \\
\hline Kester, 2016 & & & & $X$ & & & & $X$ \\
\hline Total & 7 & 2 & 2 & 5 & 1 & 5 & 1 & 3 \\
\hline
\end{tabular}

Legendas: ATC - Ataques cibernéticos / ESC - Escolaridade dos empregados, desemprego / INV - Investimentos altos, retorno do capital / IOP - Interoperabilidade, padronização na comunicação / EEL - Consumo de energia elétrica / TEC - Tecnologias diferentes de cada fornecedor, comunicação entre os equipamentos / REG Regulamentação dos dados coletados FOR - Falta de capacitação de fornecedores na cadeia produtiva.

A revisão bibliográfica realizada nas bases: Science Direct, Emerald, Compendex, Science Direct, Scopus, SAGE e Google Acadêmico, selecionou 13 artigos referentes ao tema. Dentre estes, foram identificadas 8 barreiras mostradas no Quadro 1, com destaque para as questões de segurança que foram mencionadas por sete entre os treze artigos selecionados. A segunda barreira mais citada foi a interoperabilidade, que a literatura apresenta como uma barreira à conectividade entre os objetos, que é fundamental para a implantação da | 4.0.

\section{Metodologia}

Inicialmente neste trabalho foi feita uma revisão bibliográfica sobre o tema proposto, cujos achados foram devidamente explanados no item anterior. Para verificar se a barreiras identificadas estão presentes em empresas associadas à cadeia de suprimentos da indústria automotiva no Brasil, recorreu-se a uma pesquisa de campo a seguir descrita. A estrutura metodológica desenvolvida no presente trabalho está mostrada na Figura 2. 
Figura 2 - A estrutura metodológica deste trabalho

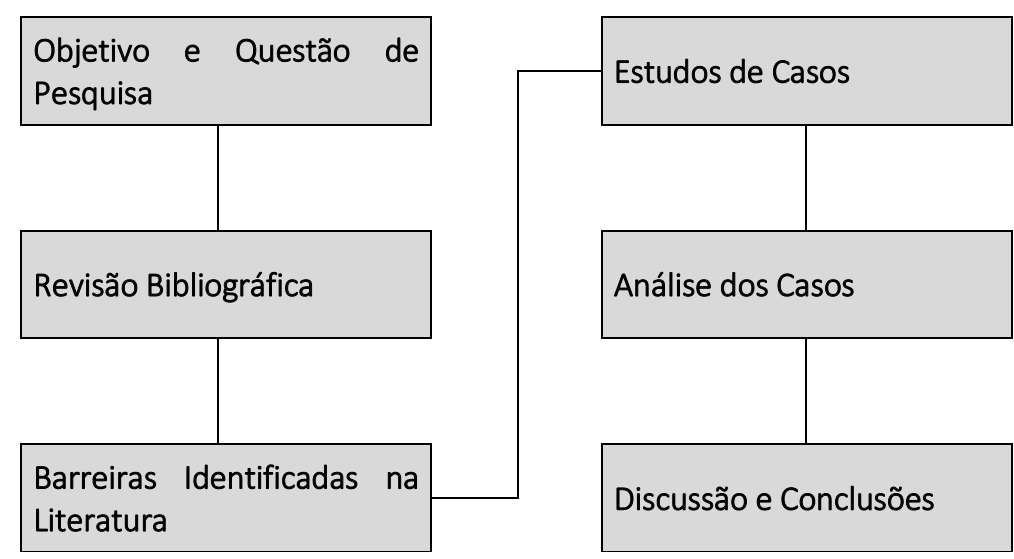

Fonte: Os autores.

De acordo com Yin (2010), para se escolher o método de pesquisa a ser adotado, uma condição primordial e importante é o tipo de questão que se busca responder. O autor indica o estudo de caso como o método de pesquisa ideal, se as questões que envolvem a identificação do tipo "como" e "porquê" certos fenômenos ocorrem. Ainda segundo Yin (2010), pode-se aplicar o estudo de caso como uma pesquisa empírica, quando esta investiga, dentro de um contexto do mundo real, um fenômeno contemporâneo no qual não haja fronteiras claras e evidentes entre o fenômeno e o contexto. De fato, o presente trabalho buscou verificar "como" as empresas e profissionais associados à indústria automotiva brasileira enxergam as barreiras à implementação da | 4.0. Além disso, nos casos estudados não se percebe claramente as fronteiras entre as barreiras e o contexto nos quais elas atuam. Assim, pelas razões mencionadas, pode-se estabelecer que o estudo de caso é o método de pesquisa mais adequado ao estudo empírico que este artigo propõe desenvolver.

Com relação à técnica de coleta de dados, deve-se considerar que os estudos de casos que se foram analisados verificaram como estava a percepção das empresas, por meio de seus executivos, com relação às barreiras da Indústria 4.0. Para tanto, na pesquisa de campo que se desenvolveu utilizou-se como técnica de coleta de dados a entrevista semiestruturada. Bryman (1995) atesta que a utilização desse tipo de entrevista deve ser a técnica de coleta de dados preferida para as pesquisas do tipo qualitativo. Segundo o autor, o seu propósito é deixar a entrevista transcorrer de modo mais natural, permitindo ao entrevistado abordar as questões em discussão, com mais tranquilidade, minimizando as restrições às suas considerações. Trata-se quase de uma conversa informal entre entrevistado e entrevistador na qual as questões relevantes para a pesquisa vão sendo desenvolvidas na medida em que a entrevista flui naturalmente. Verifica-se, assim, que esta é uma técnica conveniente às entrevistas realizadas por este trabalho, nas quais espera-se que os entrevistados possam livremente se expressar sobre as barreiras que entendem existir para a adoção da I 4.0.

Embora a na entrevista semiestruturada a conversa possa fluir naturalmente, Cauchick Miguel e Sousa (2012) recomendam que nesse tipo de entrevista o pesquisador elabore um roteiro contendo as questões que pretende abordar durante a conversa com o entrevistado. No entanto, esse roteiro é apenas um guia para o pesquisador não deixar de abordar aspectos relevantes de sua pesquisa, uma vez que ele tem a liberdade de desenvolver o tema com o entrevistado na direção que achar mais conveniente. 
Com relação ao número de estudos de caso a serem considerados, Yin (2010) apresenta duas estratégias: (1) quando os resultados assumidos são similares para os diversos casos estudados, a estratégia deveria ser a "replicação literal". Nessa condição, seria suficiente realizar o estudo de dois ou três casos; e (2) quando os resultados assumidos são contrários para os diversos casos estudados, mesmo antes da realização do estudo, a estratégia deveria ser a "replicação teórica". Nessas situações, mais de quatro casos deverão ser considerados.

O estudo empírico que se desenvolveu neste trabalho, investigou a percepção das empresas com relação às barreiras referentes a implantação da Indústria 4.0. Como pode haver, mesmo que teoricamente, alguma diferença entre as barreiras identificadas nas diferentes empresas a serem pesquisadas, optou-se por 6 estudos de casos, realizados em empresas e profissionais de alguma forma relacionados à cadeia automotiva no Brasil.

Para tanto, tomou-se o cuidado de escolher empresas com projeção no mercado automotivo nacional. Foram inicialmente selecionadas duas montadoras de automóveis e uma empresa de autopeças de grande porte. Também foi escolhida uma empresa de consultoria na área de segurança cibernética intimamente ligada à indústria automotiva e um especialista no tema, professor de uma universidade europeia, pesquisador do setor automotivo brasileiro e da Indústria 4.0. Por questões de privacidade, os nomes das empresas e das pessoas envolvidas nas entrevistas são omitidos. Assim temos para os estudos de caso:

Montadora 1 - Montadora de veículos com diversas fábricas no Brasil, instalada há mais de 90 anos. Em 2014 ela vendeu em torno de 580.000 veículos no mercado brasileiro, com uma participação de 17,4\% no mercado interno. A entrevista foi feita na fábrica instalada no Estado de São Paulo.

Montadora 2 - Montadora de veículos e caminhões, iniciou a montagem de carros no Brasil a partir da década de 20, com peças importadas. Foi a segunda filial sul americana da matriz localizada nos EEUU, tendo lançado seu primeiro automóvel fabricado no Brasil na década de 60. Em 2017 foi a quarta maior força no mercado automobilístico brasileiro. A entrevista foi realizada na planta localizada no Estado de São Paulo.

Fornecedor 1 - Empresa afiliada a um grupo industrial norte-americano, fornecedora de primeira camada na cadeia automobilística com mais de 60 anos de história no Brasil. Tem uma planta na cidade São Paulo para atender o Mercosul e conta hoje com aproximadamente 1.000 funcionários.

Consultoria 1 - Empresa especializada em segurança, que atua no território nacional com foco nas tecnologias atuais utilizadas pela Indústria 4.0, com mais de 20 anos de experiência no mercado automotivo.

Especialista 1 - Professor de uma Universidade Italiana, pesquisador e estudioso da cadeia automotiva brasileira e da indústria 4.0 .

\section{Resultados obtidos}

Os estudos de caso permitiram levantar diversas opiniões sobre as barreiras enfrentadas para a implantação da I 4.0 na cadeia automotiva brasileira. Inicialmente analisam-se os casos isoladamente e em seguida faz-se a comparação das semelhanças e dissimilaridades entre os diversos estudos.

\subsection{Análise dos casos}

Na Montadora 1 foi feita a entrevista com a participação dos Gerentes de TI, Engenharia Industrial, Manutenção e o Diretor Industrial. Eles foram unânimes em dizer que a indústria automobilística do Brasil ainda 
está longe da Indústria 4.0, porém esse é o caminho que perseguem uma vez que essa realidade seria irreversível, já existindo estudos avançados nesta área. Apesar de tal afirmação, essa montadora já possui dezenas de robôs controlados remotamente por VPN (Rede Privada Virtual). Atualmente ainda não utilizam a loT e estão desenvolvendo um projeto de máquinas inteligentes.

Dentre as barreiras apontadas pelos entrevistados foram citadas com mais ênfase, não em ordem de prioridade:

- Maior vulnerabilidade a ataques cibernéticos - o fato de haver muitas portas de entrada, devido ao uso crescente de smartphones e tablets conectados na rede todo o sistema fica mais vulnerável;

- Instabilidade no fornecimento de energia elétrica - No Brasil mesmo empresas que possuem cabines primárias e geradores, como é o caso desta montadora, correm o risco de ficar períodos sem energia elétrica devido ao baixo investimento em infraestrutura nos últimos anos no país;

- Precariedade nas estruturas tecnológicas dos fornecedores - Os investimentos são significativos nestas novas tecnologias o que impede que muitos fornecedores da cadeia de suprimentos, em vários níveis, nem sempre consigam acompanhar a evolução tecnológica necessária;

- Muitas operações manuais - a própria montadora reconhece que ainda há muitas operações manuais o que dificultaria que muitas tecnologias da I 4.0 sejam implantadas.

Na Montadora 2 foi feita a entrevista com o responsável pelo desenvolvimento do projeto da Indústria 4.0. O entrevistado também entende que a cadeia de suprimentos da indústria automotiva brasileira ainda não tem a Industria 4.0 implementada, mas coloca esta revolução como uma oportunidade para igualar os países desenvolvidos como os em desenvolvimento, por se tratar de conceitos para os quais estão todos partindo do zero. Ele entende que dentro da Indústria 4.0 a montadora deixará de ser o centro da cadeia de suprimentos, para passar a ser somente um elo. Nesta empresa, os estudos sobre a Indústria 4.0 estão bem evoluídos e este é considerado como um caminho sem volta. Segundo o entrevistado, uma das grandes vantagens da | 4.0 está no fato de que eles passariam de uma rastreabilidade de 10\% para o recall, para $100 \%$, já que as novas tecnologias da I 4.0 permitem tal melhoria por meio de dados empíricos obtidos em uma fábrica virtual, chamada planta gêmea. Segundo a opinião do entrevistado, as principais barreiras a serem enfrentadas na implementação da | 4.0 seriam, não em ordem de prioridade:

- Falta de uniformidade nos protocolos de comunicação entre as máquinas e com os sensores - A linguagem unificada na comunicação entre objetos é fundamental para que haja a conectividade e a interoperabilidade necessária na Indústria 4.0. Portanto a uniformidade nos protocolos de comunicação entre os objetos, na visão do entrevistado, ainda é um obstáculo significativo na evolução da Indústria 4.0;

- Baixa escolaridade da mão de obra, implicando em desemprego - Nossa capacidade de formar engenheiros e técnicos ainda é muita baixa se comparada com os países desenvolvidos. Portanto, ainda 
existe uma mão de obra operacional sem a devida capacitação para operar equipamentos com as novas tecnologias;

- Maior possibilidade de ataques cibernéticos - o fato de haver muitas portas de entrada, devido ao uso crescente de smartphones e tablets conectados na rede todo o sistema acaba se tornando mais vulnerável;

- Altos investimentos - Apesar de ter havido uma diminuição de preços em alguns produtos, como por exemplo sensores, ainda a maior parte dos equipamentos e tecnologias necessárias para a completa utilização da I 4.0 é importada e vem com um alto valor agregado o que torna os investimentos na Indústria 4.0 significativos para as empresas de países em desenvolvimento como o Brasil

No Fornecedor 1 foi feita a entrevista com três Gerentes: o de Engenharia de Processo, o de Qualidade e Fornecedor e o de TI. Quanto ao desenvolvimento da Indústria 4.0, percebe-se um nível abaixo das montadoras. Apesar de terem a noção e o conhecimento do que se trata a I 4.0, ficam sob a tutela do que a montadora exige. Assim, por conta de algumas exigências de seus clientes, a empresa já possui rezoável tecnologia embarcada, porém longe ainda de algo semelhante à Industria 4.0. Especificamente com relação à segurança, essa empresa já possui várias tratativas para prover um ambiente seguro para a produção. Resumidamente, existe um firewall, antivírus, atualizações diárias de segurança, bloqueio de pen drive, WiFi seguro, registro de logs, monitoramento e autenticação de acesso. Ainda assim, se um cyber ataque acontecer, existe um plano de recuperação com backups de todos ambientes e também pode-se bloquear o acesso externo por meio do firewall e preservar a produção. Como barreiras os entrevistados destacaram, não na ordem de prioridade:

- Volume de produção inconstante - Como fornecedores de primeira camada da indústria automobilística eles ficam muito suscetíveis aos programas de produção solicitados pelas montadoras, que em período de crise se tornam extremamente inconstantes, o que dificulta um planejamento;

- Instabilidade econômica - Esta é uma característica comum no país que sofre com a inconstância no mercado de commodities. Isto deixa as empresas inseguras com relação a qualquer investimento de longo prazo;

- Ataques cibernéticos - repetindo as preocupações já manifestadas pelos entrevistados de outras empresas, aqui também há o consenso que em decorrência de haver muitas portas de entrada na rede o sistema fica mais vulnerável a acessos indevidos;

- Desemprego - A automação e digitalização que existem na | 4.0, em um primeiro momento deve provocar a dispensa de mão de obra, principalmente a operacional com baixa escolaridade e pouco conhecimento tecnológico.

Na Consultoria 1 foi entrevistado o consultor sênior da empresa, que relatou que o processo tecnológico no Brasil, principalmente no que se refere à Indústria 4.0, ainda está atrasado quando comparado aos países mais desenvolvidos. A indústria brasileira ainda não se atentou para a importância de digitalizar seus processos e informatizar de maneira séria seus principais ativos, entendendo que a integração não é somente necessária, mas 
sim fundamental para que a indústria possa agregar valor em suas entregas. O foco dado pelo entrevistado sobre a I 4.0 concentrou-se principalmente no aspecto da segurança.

Segundo o consultor, quando se parte para uma integração dos pontos produtivos, acaba-se criando brechas de segurança que, se não forem tratadas, podem representar vulnerabilidades sérias. Como exemplo citou o uso de tablets nos processos de controle. Se este uso não for muito bem controlado por meio de Políticas e Controles de Segurança, esse dispositivo pode representar um ponto de alta vulnerabilidade, pois estará conectado à rede da empresa e com acesso direto às maquinas operacionais. Um outro risco a que esta indústria informatizada está suscetível é um ataque chamado SCADA. Os ambientes SCADA (Supervisory Control and Data Acquisition ou Controle de Supervisão e Aquisição de Dados) consistem em sistemas para controle industrial, implementados em grande escala, que têm como função principal monitorar, gerenciar e administrar infraestruturas críticas em diversos segmentos como transporte, energia nuclear, energia elétrica, gás, água etc. Ataques SCADA são ataques que atingem os periféricos que controlam os monitores de equipamentos críticos que se aproveitam das vulnerabilidades para iniciar um ataque. Como exemplo de um ataque deste tipo o entrevistado mencionou o acontecido na Ucrânia em dezembro de 2016, que paralisou totalmente uma usina geradora de energia elétrica.

Ainda segundo o consultor, pode-se perceber a fragilidade dos sistemas pelos recentes ataques do WannaCry e o Petya, nos quais várias organizações, ao redor do mundo, foram atacadas e s tiveram sérios problemas para restabelecer suas operações. Isso denota a dificuldade que se tem de trabalhar com Planos de Continuidade de Negócios (PCN), visto pela grande maioria dos empresários como um custo e não como uma necessidade de sobrevivência da empresa em caso de um desastre. Atualmente, ataques cibernéticos podem ser muito mais destrutivos que um desastre natural. Adotar políticas de segurança compatíveis, manter versões atualizadas dos softwares, não trabalhar com softwares piratas e manter controle sobre os periféricos em uso pelos usuários (principalmente na chamada loT), são recomendações que todas as empresas deveriam observar nessa conjuntura de digitalização dos processos produtivos. Segundo o entrevistado, a função da área de atuação da consultoria deveria se restringir à segurança. Por consequência, os ataques cibernéticos foi a única barreira elencada pelo consultor.

O Especialista 1 com base nas suas pesquisas tanto acadêmicas em empresas automotivas europeias e brasileiras, elencou as seguintes barreiras para implantação da Indústria 4.0, não em ordem de prioridade.

- Necessidade de padronização nas comunicações, devido a interoperabilidade da Indústria 4.0 - Ainda falta uma padronização nos protocolos de comunicação entre os fabricantes de equipamentos e entre as indústrias, pois todos querem que prevaleça o seu próprio protocolo;

- Cyber ataques na infraestrutura das empresas- Os chamados ataques cibernéticos aos periféricos, onde são mais vulneráveis podem trazer muitos problemas para a empresa, mesmo que o ataque não ultrapasse todas as camadas e não atinja o coração do sistema;

- Regulamentação para proteção dos dados coletados pela empresa - Falta uma regulamentação clara que proteja os usuários da internet. Como os dados coletados ficam na nuvem, muitas organizações sentem-se inseguras em utilizar esses serviços; 
- Necessidade de aproveitamento dos equipamentos antigos face às novas tecnologias incorporadas nas novas máquinas - Muitos equipamentos ainda se encontram em perfeito funcionamento, porém não estão adaptados para receberem as novas tecnologias, isto implica em um investimento que nem todas as organizações estão dispostas a fazer. Em outras situações, os equipamentos até já são digitalizados, mas a diferença de protocolos de comunicação acaba se tornando em um problema não facilmente superável.

- Retorno do capital empregado (ROI), face aos altos investimentos envolvidos na implantação da indústria 4.0 - O ROI é de longo prazo, o que não agrada investidores quando se considera em um país que vive uma instabilidade econômica significativa como o Brasil está vivendo atualmente;

- Mudança nas habilidades dos colaboradores e necessidade das escolas se adaptarem à formação deste novo profissional - As competências dos colaboradores devem se adaptar às novas necessidades e nisto a academia tem um papel fundamental na formação de novos profissionais aptos a enfrentarem este desafio, onde novas profissões surgiram nos próximos anos.

\subsection{Comparação dos casos}

Com base nos estudos de caso foram identificadas nove barreiras à implantação da | 4.0, conforme mostra o Quadro 2. Nota-se que as questões referentes aos ataques cibernéticas foram citadas pela unanimidade dos entrevistados, com destaque também para as questões referentes ao nível de investimentos e ao desemprego, o que demonstra uma preocupação de todos com as questões sociais, o que também vem ao encontro das preocupações atuais com a situação econômica que o país atravessa.

Quadro 2 - Barreiras verificadas nos Estudo de Casos

\begin{tabular}{|l|l|l|l|l|l|l|l|l|l|}
\hline Casos & ATC & ESC & INV & IOP & ECO & EEL & TEC & FOR & REG \\
\hline Montadora 1 & $\mathrm{X}$ & & $\mathrm{X}$ & & & $\mathrm{X}$ & $\mathrm{X}$ & $\mathrm{X}$ & \\
\hline Montadora 2 & $\mathrm{X}$ & $\mathrm{X}$ & $\mathrm{X}$ & $\mathrm{X}$ & & & $\mathrm{X}$ & & \\
\hline Fornecedor 1 & $\mathrm{X}$ & $\mathrm{X}$ & & & $\mathrm{X}$ & & & & \\
\hline Consultor 1 & $\mathrm{X}$ & & & & & & $\mathrm{X}$ & & \\
\hline Especialista 1 & $\mathrm{X}$ & $\mathrm{X}$ & $\mathrm{X}$ & $\mathrm{X}$ & & $\mathrm{X}$ & & & $\mathrm{X}$ \\
\hline
\end{tabular}

Legendas: ATC - Ataques cibernéticos / ESC - Escolaridade dos empregados, desemprego / INV Investimentos altos, retorno do capital / IOP - Interoperabilidade, padronização na comunicação / EEL - Consumo de energia elétrica / TEC - Tecnologias diferentes de cada fornecedor, comunicação entre os equipamentos / REG - Regulamentação dos dados coletados FOR - Falta de capacitação de fornecedores na cadeia produtiva.

Fonte: Os autores.

\section{Discussão}

Cruzando os dados obtidos na revisão de literatura com os estudos de caso, cujos dados estão sintetizados nos Quadros 1 e 2, respectivamente, pode-se notar que a tanto a literatura como os estudos de casos feitos identificaram praticamente as mesmas barreiras à implementação da | 4.0. No entanto, a percepção dos entrevistados no país divergem quanto à importância relativa de cada barreira. Enquanto há unanimidade tanto 
na literatura como na prática de que o risco de ataques cibernéticos e as dificuldades representadas pelas diferentes tecnologias utilizadas pelos fornecedores são barreiras relevantes, a padronização dos protocolos de comunicação tem destaque na literatura, mas não obtiveram igual relevância nos estudos de caso, talvez refletindo o fato de que tal barreira ainda não tenha sido devidamente percebida em razão do estágio atual da implementação da I 4.0 no Brasil . Por outro lado, barreiras como escolaridade dos empregados / desemprego e necessidade de investimento mostraram-se importantes nos estudos realizados e não receberam igual relevância na literatura, muito provavelmente como decorrência da baixa escolaridade e formação dos operadores locais, realidade muito diferente nos países desenvolvidos nos quais a | 4.0 tem avançado mais celeremente. Tais divergências justificam-se pelo fato da literatura analisada ter sido composta somente por autores estrangeiros (por inexistência de nacionais relevantes). É de se esperar que as percepções das empresas localizadas no Brasil, onde a realidade da I 4.0 é muito diferente, que tal situação se reflita na opinião dos entrevistados.

\section{Conclusão}

A análise dos estudos não tem divergências significativas com a revisão da literatura e em ambos os casos a principal barreira elencada é a questão da segurança, nitidamente pode-se perceber uma preocupação dos gestores com relação à fragilidade das empresas face aos cybers ataques que vêm afligindo várias plantas ao redor do mundo.

Este artigo contribui para mostrar que ainda existe uma insegurança com relação a implantação da | 4.0 pelas empresas, seja por problemas de cyber segurança, como pelos investimentos envolvidos.

Outras barreiras significativas são a interconexão entre os diversos equipamentos e a uniformidade dos protocolos utilizados no diálogo entre os equipamentos interconectados.

Este trabalho traz também contribuições tanto para a teoria como para a prática. Para o campo do conhecimento da Engenharia de Produção, este é um artigo que explora questões relativas à implantação da Indústria 4.0 no Brasil, o que auxilia no reforço de um arcabouço teórico sobre esse tema em países em desenvolvimento como o Brasil. Do ponto de vista prático, os resultados aqui mostrados auxiliarão os executivos e profissionais das indústrias brasileiras a começar a entender as barreiras e dificuldades a serem enfrentadas na implantação da | 4.0, com o objetivo de prepararem-se adequadamente para superar as barreiras impostas dessa adoção.

Como toda pesquisa, esta também tem limitações. A primeira reside no fato de que as conclusões aqui mostradas foram obtidas por meio de estudo de casos, o que não permite uma generalização dos resultados alcançados. Como também os casos estudados focaram a cadeia automotiva brasileira, não se pode inferir que as mesmas barreiras sejam identificadas em outros segmentos industriais. Ainda, o trabalho propôs-se apenas a identificar as barreiras relevantes para a adoção da I 4.0 e não avaliar ou propor ações para superá-las. Por isso, sugere-se que pesquisas futuras sejam realizadas com o foco de ampliar as avaliações aqui feitas de modo a poder obter conclusões mais amplas e generalizáveis sobre o tema estudado, avaliando ainda alternativas estratégicas, gerenciais e operacionais para a adequada superação das barreiras identificadas. 
Ahmeda, E., Yaqooba, I., Hashema, I.A.T., Khanb, I., Ahmeda ,A.I.A.; Imranc, M., \& Vasilakos, A.V. (2017) The role of big data analytics in Internet of Things. Computer Networks, 129(2), 459-471.

Akinlar, S. (2014) Logistics 4.0 and challenges for the supply chain planning and IT. Dortmund, Germany: Fraunhofer IML.

Bauters, K., Cottyn, J., Claeys, D., Slembrouck, M., Veelaert, P., \& Landeghem. H.V. (2018) Automated work cycle classification and performance measurement for manual work stations. Robotics and Computer-Integrated Manufacturing, 51, June 2018, 139-157.

Berger, R. (2014) Industry 4.0: The new industrial revolution - How Europe will succeed, Frankfurt, Germany: Roland Berger Strategy Consultants.

Bryman, A. (1989) Research methods and organization studies. London, UK: Unwin Hyman.

Buhr, D. (2015) Industry 4.0 - New tasks for innovation policy. Bonn, Germany: Friedrich-Ebert-Stiftung.

Cauchick Miguel, P.A., \& Sousa, R. O método do estudo de caso na Engenharia de Produção In Cauchick Miguel, P.A. (Org.) (2012) Metodologia de pesquisa em Engenharia de Produção e Gestão de Operações. 2.ed. Rio de Janeiro: Elsevier.

CNI - Confederação Nacional da Indústria. (2016) Sondagem Especial 66 - Indústria 4.0: novo desafio para a indústria brasileira. Brasília, Brasil: CNI.

Dilberoglu, U.M., Gharehpapagh, B., Yaman, U., \& Dolen, M. (2017) The role of additive manufacturing in the era of industry 4.0. Procedia Manufacturing, 11, 545-554.

Dopico, M. et al. (2016) A vision of industry 4.0 from an artificial intelligence point of view. In: Proceedings on the International Conference on Artificial Intelligence, ICAI -2016, Las vegas, Nevada, USA: ICAI, 407-413.

Gabriel, M., \& Pessl, E. (2016) Industry 4.0 and sustainability impacts : critical discussion of sustainability aspects with a special focus. International Journal of Engineering, 14(2), 131-137.

Hannah, M. (2015) Industry 4.0 : turning the vision into reality. Manufacturing Business Technology, 72, April, 5-9.

IPESI. (2016) Indústria 4.0 faz aumentar risco de ataques cibernéticos contra os sistemas de controle industrial. Ipesi, 2016. Disponível em http://ipesi.com.br/Noticias/4362-industria-40-faz-aumentar-risco-de-ataquesciberneticos-contra-os-sistemas-de-controle-industrial, acessso em 21 jun 2018.

Khan, A., \& Turowski, K. (2016) A Perspective on Industry 4.0: From Challenges to Opportunities in Production Systems. In: Proceedings of the international conference on internet of things and big data - IOTBD 2016, Rome: IOTBD, 441-448.

Kester, R. (2016) Demystifying the internet of things: industry impact, standardization problems, and legal considerations. Elon, NC: Elon University. Disponível em: www.elon.edu/e/CmsFile/GetFile?FileID=447. Acesso em: 27 Abr 2018.

Lasi, H., Fettke, P., Kemper, H. G., Feld, T., \& Hoffmann, M. (2014) Industry 4.0. Business \& Information Systems Engineering, 6(4), 239-242.

Lucato, W.C., Vieira Júnior, M., Vanalle, R.M., \& Salles, J.A.A. (2012) Model to measure the degree of competitiveness for auto parts manufacturing companies. International Journal of Production Research, 50(19), 5508-5522.

Li, B., \& Li, Y. (2017) Internet of things drives supply chain innovation: a research framework. The International Journal of Organizational Innovation 9(3), 71-93.

Marconi, M.A., \& Lakatos, E.M. (2010) Fundamentos de metodologia científica. 7. ed. São Paulo, SP, Atlas.

Marques, F. (2017) O Brasil da Internet das Coisas. Pesquisa FAPESP, 259, 19-27.

Matt, D. (2014) Smart factory: industria del futuro: esempi di best practice. Milano: Fraunhofer Italia.

Mosterman, P.J., \& Zander, J. (2015) Industry 4.0 as a Cyber-Physical System study. Software \& Systems Modeling, 15(1), 17-29.

Ng, I.C L., \& Wakenshaw, S.Y.L. (2017) The Internet-of-Things: Review and research directions. International Journal of Research in Marketing, 34(1), 3-21.

Ochoa, S. F., Fortino,G., \& Fatta, G. (2017) Cyber-physical systems, internet of things and big data. Future Generation Computer Systems, 75, 82-84.

Peters, S., Lanza, G., Jun, N., \& Xiaoning, j. (2014) Automotive manufacturing technologies - an international viewpoint. Manufacturing Review, 1(10), 1-12.

Mendes, C.R., Siemon, F.B., \& Campos, M.M. (2017),. Estudos de caso da indústria 4.0 aplicados em uma empresa automobilística. POSGERE, 1(4), 15-25.

Schwab. K. (2016) A Quarta Revolução Industrial. São Paulo, SP: Edipro.

Siemens. O Brasil está preparado para a indústria 4.0. Revista Exame, São Paulo 16 maio 2016. Disponível em: http://exame.abril.com.br/tecnologia/o-brasil-esta-pronto-para-a-industria-4-0. Acesso em: 14/09/2017

Tupa, J., Simota, J., \& Steiner, F. (2017) Aspects of risk management implementation for Industry 4.0. Procedia Manufacturing, 11, $1223-1230$. 
Valverde, R. et al. (2015) The effectiveness of COBIT 5 Information Security Framework for reducing Cyber of Attacks on Supply Management System. IFAC- PapersOnLine, 48(3), 1846-1852..

Vanalle, R.M.; Salles, J.A.A. (2011) Relação entre montadoras e fornecedores: modelos teóricos e estudos de caso na indústria automobilística brasileira. Gestão \& Produção, 18(2), 237-250.

Veza, I.. Mladineo, M., \& Gjeldum, N. (2016) Selection of the basic lean tools for development of Croatian model of innovative smart enterprise. Tehnički vjesnik, 23(5), 1317-1324.

$\mathrm{Xu}$, L.D., He, W., \& Li, S. (2014) Internet of Things in Industries: A Survey. IEEE Transactions on Industrial Informatics, 10(4), 2233-2243.

YIN, R.Y. Estudo de caso - planejamento e métodos. 4. ed. Porto Alegre: Bookman, 2010.

Weber, R. H., Studer, E. (2016) Cybersecurity in the Internet of Things: Legal aspects. Computer Law \& Security Review, 32(5), 715-728.

Witkowski, K. (2017) Internet of Things, Big Data, Industry 4.0 - Innovative Solutions in Logistics and Supply Chains Management. Procedia Engineering, 182, 763-769.

Wylie, D. (2016) Pay attention: Industrie 4.0 and ICS cyber security. Control Engineering, 4, 1-7.

Recebido em: 25 set. 2018 / Aprovado em: 23 jan. 2019

Para referenciar este texto

Pacchini, A. P. T., Santos, J. C. da S., Logiudice, R., \& Lucato, W. C. (2020). Indústria 4.0: barreiras para implantação na indústria brasileira. Exacta, 18(2), 278-292. https://doi.org/10.5585/ExactaEP.v18n2.10605. 